\title{
A clinical comparison of SIDS and explained sudden infant deaths: how healthy and how normal?
}

Martin Ward Platt, Peter S Blair, Peter J Fleming, Iain J Smith, Tim J Cole, Charlotte E A Leach, P J Berry, Jean Golding, and the CESDI SUDI Research Group

\begin{abstract}
Objectives-To compare the clinical characteristics associated with sudden infant death syndrome (SIDS) and explained sudden unexpected deaths in infancy (SUDI).

Design-Three year population based, case control study with parental interviews for each death and four age matched controls.

Setting-Five regions in England (population, > 17 million; live births, > 470000$)$.

Subjects-SIDS: 325 infants; explained SUDI: 72 infants; controls: 1588 infants.

Results-In the univariate analysis, all the clinical features and health markers at birth, after discharge from hospital, during life, and shortly before death, significant among the infants with SIDS were in the same direction among the infants who died of explained SUDI. In the multivariate analysis, at least one apparent life threatening event had been experienced by more of the infants who died than in controls (SIDS: $12 \% v 3 \%$ controls; odds ratio $(O R)=2.55 ; 95 \%$ confidence interval (CI), 1.02 to 6.41; explained SUDI: $15 \% v$ $4 \%$ controls; $O R=16.81 ; 95 \%$ CI, 2.52 to 112.30). Using a retrospective illness scoring system based on "Baby Check", both index groups showed significant markers of illness in the last 24 hours (SIDS: $22 \% v$ $8 \%$ controls; $\mathrm{OR}=4.17 ; 95 \% \mathrm{CI}, 1.88$ to 9.24; explained SUDI: $49 \%$ v $8 \%$ controls; OR $=31.20 ; 95 \%$ CI, 6.93 to 140.5 ).
\end{abstract}

Conclusions-The clinical characteristics of SIDS and explained SUDI are similar. Baby Check might help identify seriously ill babies at risk of sudden death, particularly in high risk infants.

(Arch Dis Child 2000;82:98-106)

Keywords: signs of illness in infants; sudden unexpected death in infancy; sudden infant death syndrome; Baby Check

After the success of the "back to sleep" campaign and the consequent reduction in the rate of sudden infant death syndrome (SIDS), it has become apparent that the epidemiological background of those infants who now die as a result of SIDS has changed. Infants who die of SIDS tend to come from more disadvantaged backgrounds than previously, and the seasonal pattern of deaths has been attenuated. ${ }^{1}$ At the same time, cases of sudden and unexpected deaths in infancy (SUDI) that can be explained now account for a greater proportion of all such deaths (approximately $20 \%){ }^{2}$ Greater attention will have to be paid to this group of infants if there is to be any further substantial reduction in infant mortality.

The concept of "causes" of SIDS (and explained SUDI) is inadequate. Defining physiological, environmental, and social factors that tend to promote vulnerability to sudden death from any cause might be more useful. Furthermore, some of these factors might be amenable to change, whereas others might not. This could have implications for the more refined targeting of resources, support, and advice, either at the level of populations or for individual families.

Some features characteristic of SIDS suggest that vulnerability starts before birth, that it might also arise in association with factors in the neonatal period, and that it might be related to factors early in infancy. These include a higher prevalence of multiple births, lower birth weight, and shorter gestational age. Studies conducted since the fall in SIDS rates in the early 1990s confirm these characteristics and indicate maternal smoking during pregnancy as an independent and possibly causal risk factor. ${ }^{3-5}$ A prospective cohort study in Tasmania in the 1980s found that significantly more infants who died of SIDS had a long term history of illness, particularly relating to respiratory conditions. ${ }^{6}$ Studies have reported a greater frequency of hospital admissions among the infants who died as a result of SIDS, ${ }^{7-9}$ along with more attendances at the doctor's surgery and outpatient clinic in the weeks before death. ${ }^{710}$ In a retrospective study of symptoms and signs in infants dying suddenly and unexpectedly in Avon, ${ }^{11} 8 \%$ of infants who died, particularly those who died of infections, had symptoms suggesting they had been severely ill in the 24 hours preceding their deaths. Recognition and appropriate action in response to such potentially important findings, either by parents or by health care professionals, might reduce the risk of death for such infants. However, the value of these observations might be limited by recall bias, although the evidence of increased contact with health professionals just before death suggests differences in parental recall cannot wholly explain differences in infant illness. 
The study of sudden unexpected deaths in infancy (part of the confidential enquiry into stillbirths and deaths in infancy: CESDI SUDI study) was designed to elicit information on symptoms and signs before death using a study design that minimised recall bias. One of the aims was to investigate which clinical features and health indicators at birth, after discharge from hospital, during postnatal life, and shortly before death are now most closely associated with sudden unexpected death, including SIDS. The aim was to compare the findings for SIDS with those for infants who died of explained SUDI, and to evaluate whether recognition of signs or symptoms of illness could have led to earlier recognition of the infants' illnesses, and thus the potential for prevention of these deaths.

\section{Methods}

The CESDI SUDI study has been described previously. ${ }^{32-14}$ Briefly, it was a large population based, case control study conducted over a three year period from 1993 to 1996 . The study aimed to include all sudden unexpected deaths of infants aged 7-364 days from a total study population of 17.7 million. Data were collected using a standard questionnaire, by research interviewers and from medical records. Bereaved families were visited within days of the death for a narrative account and a second visit within two weeks to complete a detailed questionnaire. There were four age matched controls for each case. They were visited within two weeks of the death to collect the same data as for the index case. A period of sleep (the "reference sleep") was identified in the control infant's life in the 24 hours before the interview, corresponding to the time of day during which the index baby had died.

The questionnaire included demographic and social data; the medical history of the infant and other family members; use of cigarettes, alcohol, and drugs; the precise sleeping arrangements for the infant; full details of the events preceding and the circumstances surrounding the death. There were several questions on the parents' perceptions of the infants' state of health generally, in the last week, the last 24 hours, and immediately before death or final sleep. A modified form of the Cambridge "Baby Check" questionnaire (appendix), which we have validated previously for studies of infant deaths ${ }^{11}{ }^{15}$ was included, with questions referring to the final 24 hours before death or reference sleep. Baby Check is a system to help parents and doctors quantify illness in babies during the first 6 months of life (which includes over $80 \%$ of the babies in our study). It is based on seven symptoms and 12 signs, each of which receives a score if present: the higher the score the less well the baby. For all but four questions information could be gathered retrospectively. Parental recall of fever was used as a proxy measure for a temperature measurement. The scores are grouped and advice to parents is linked to each group:

(1) Score 0 to 7: your baby is entirely well or only a little unwell, and medical attention should not be necessary.
(2) Score 8 to 12: your baby is unwell but unlikely to be seriously ill. You might want advice from your doctor, health visitor, or midwife.

(3) Score 13 to 19: your baby is ill. Contact your doctor and arrange for your baby to be seen.

(4) Score 20+: your baby is seriously ill and should be seen by a doctor straight away.

The maximum score possible using the Baby Check is 111 , the maximum possible score using the modified Baby Check used in the questionnaire was 96 (appendix).

ESTABLISHING THE CAUSE OF DEATH

Because all deaths were unexpected a postmortem examination was required routinely by the coroner, who might instruct a paediatric pathologist, a forensic pathologist, or a general pathologist according to the circumstances. All pathologists carrying out necropsies were given the limited background history of the SUDI cases, collected by the coroner, and were asked to follow a postmortem protocol based upon the recommendations of the Royal College of Pathologists. ${ }^{16}$ Cause of death was established by a multidisciplinary committee, which always included a general practitioner, a health visitor, an obstetrician or a midwife, a paediatrician, a paediatric pathologist, and a public health doctor. Confidentiality was maintained by anonymising all papers, and by ensuring that no panel member had previous knowledge of the case in question. To improve consistency, the chairs for the panels in each region were permanent, and other panel members were drawn from a small pool of experienced practitioners in each speciality. The research interviewer was not present at the panel but contributed to the documentation. To ensure reproducibility and consistency, a subset of cases were subject to assessment on more than one occasion.

All deaths were classified according to the Avon clinicopathological system. ${ }^{13}{ }^{17}$ SIDS was defined as the sudden death of an infant, unexpected by history, for which no sufficient explanation was identified by the multidisciplinary panel after a full paediatric necropsy, review of the medical and social history, and assessment of the circumstances of the death. Explained sudden unexpected deaths in infancy included: (1) deaths occurring in the course of an acute illness that was not recognised by carers and/or by health professionals as potentially life threatening; (2) deaths occurring in the course of a sudden acute illness of less than 24 hours duration in a previously healthy infant, or a death that occurred after this if intensive care had been instituted within 24 hours of the onset of the illness; (3) deaths arising from a pre-existing condition that had not been recognised previously by health professionals; (4) deaths resulting from any form of accident, trauma, or poisoning.

STATISTICAL METHODOLOGY

Non-parametric distributions were described using medians and interquartile ranges. Odds 
Table 1 Univariate findings for clinical characteristics of SIDS cases and control infants

\begin{tabular}{|c|c|c|c|c|c|c|}
\hline & \multicolumn{2}{|l|}{ SIDS } & \multicolumn{2}{|l|}{ Controls } & \multirow[b]{2}{*}{$O R(95 \% C I)$} & \multirow[b]{2}{*}{$p$ Value } \\
\hline & $n$ & $\%$ & $n$ & $\%$ & & \\
\hline \multicolumn{7}{|l|}{ At birth } \\
\hline$<10$ th birth weight centile & $50 / 323$ & 15.5 & $99 / 1286$ & 7.7 & $2.27(1.50$ to 3.45$)$ & $<0.0001$ \\
\hline$<37$ weeks' gestation & $63 / 323$ & 19.5 & $70 / 1288$ & 5.4 & $3.44(2.47$ to 4.79$)$ & $<0.0001$ \\
\hline Twin or triplet & $17 / 325$ & 5.2 & $12 / 1300$ & 0.9 & 8.27 (3.41 to 20.05$)$ & $<0.0001$ \\
\hline Resuscitation required & $25 / 321$ & 7.8 & $24 / 1281$ & 1.9 & $4.11(2.13$ to 7.92$)$ & $<0.0001$ \\
\hline Admission to SCBU & $80 / 323$ & 24.8 & $92 / 1291$ & 7.1 & $4.25(2.91$ to 6.21$)$ & $<0.0001$ \\
\hline Neonatal problem noted & $83 / 322$ & 25.8 & $143 / 1291$ & 11.1 & $2.72(1.93$ to 3.83$)$ & $<0.0001$ \\
\hline Major congenital anomaly & $16 / 323$ & 5.0 & $27 / 1290$ & 2.1 & $2.46(1.20$ to 5.07$)$ & 0.01 \\
\hline Home delivery & $7 / 318$ & 2.2 & $21 / 1279$ & 1.6 & $1.34(0.48$ to 3.31$)$ & 0.67 \\
\hline \multicolumn{7}{|l|}{ After hospital discharge } \\
\hline On vitamin supplements & $24 / 323$ & 7.4 & $20 / 1299$ & 1.5 & $5.18(2.58$ to 10.40$)$ & $<0.0001$ \\
\hline Problem with regurgitation ${ }^{\star}$ & $30 / 316$ & 9.5 & $48 / 1294$ & 3.7 & $2.87(1.68$ to 4.90$)$ & $<0.0001$ \\
\hline Problem with colic ${ }^{\star}$ & $39 / 317$ & 12.3 & $113 / 1297$ & 8.7 & $1.59(1.03$ to 2.44$)$ & 0.03 \\
\hline Problem with weight gain ${ }^{\star}$ & $38 / 314$ & 12.1 & $101 / 1297$ & 7.8 & $1.67(1.08$ to 2.59$)$ & 0.02 \\
\hline Problem with illness ${ }^{\star}+$ & $60 / 315$ & 19.0 & $156 / 1296$ & 12.0 & $1.75(1.21$ to 2.55$)$ & 0.003 \\
\hline Problem with crying ${ }^{\star}$ & $28 / 315$ & 8.9 & $155 / 1297$ & 12.0 & $0.72[0.46-1.12]$ & 0.15 \\
\hline Problem with sleeping ${ }^{\star}$ & $24 / 315$ & 7.6 & $121 / 1297$ & 9.3 & $0.80(0.49$ to 1.29$)$ & 0.40 \\
\hline Problem with feeding ${ }^{\star}$ & $55 / 316$ & 17.4 & $203 / 1297$ & 15.7 & $1.14(0.81$ to 1.59$)$ & 0.50 \\
\hline \multicolumn{7}{|l|}{ During life } \\
\hline Episode of convulsions ${ }^{\star}$ & $11 / 318$ & 3.5 & $12 / 1299$ & 0.9 & $4.49(1.80$ to 11.17$)$ & 0.001 \\
\hline Episode of lifelessness ${ }^{\star}$ & $37 / 317$ & 11.7 & $39 / 1299$ & 3.0 & $5.39(3.11$ to 9.34$)$ & $<0.0001$ \\
\hline Hospital admission ${ }^{\star}$ & $84 / 321$ & 26.2 & $204 / 1299$ & 15.7 & $1.98(1.43$ to 2.74$)$ & $<0.0001$ \\
\hline \multicolumn{7}{|l|}{ Before death/reference sleep } \\
\hline Illness in last week & $84 / 322$ & 26.1 & $228 / 1299$ & 17.6 & $1.64(1.19$ to 2.24$)$ & 0.002 \\
\hline Health poor in last week ${ }^{\star}$ & $25 / 324$ & 7.7 & $52 / 1298$ & 4.0 & $2.01(1.16$ to 3.47$)$ & 0.01 \\
\hline Medication in the last week & $176 / 323$ & 54.5 & $653 / 1299$ & 50.3 & $1.17(0.91$ to 1.50$)$ & 0.23 \\
\hline Last 24 hours: Baby Check $>7^{\star}$ & $68 / 318$ & 21.4 & $97 / 1299$ & 7.5 & $4.18(2.73$ to 6.19$)$ & $<0.0001$ \\
\hline Health poor ${ }^{\star}$ & $19 / 321$ & 5.9 & $20 / 1299$ & 1.5 & $4.28(2.06$ to 8.92$)$ & $<0.0001$ \\
\hline Sweatier than usual* & $23 / 317$ & 7.3 & $52 / 1298$ & 4.0 & $2.10(1.18$ to 3.72$)$ & 0.01 \\
\hline Fed less frequently^ & $42 / 317$ & 13.2 & $67 / 1298$ & 5.2 & $3.01(1.89$ to 4.78$)$ & $<0.0001$ \\
\hline Demanded less feed* & $29 / 317$ & 9.1 & $20 / 1298$ & 1.5 & $6.44(3.19$ to 12.98$)$ & $<0.0001$ \\
\hline
\end{tabular}

Centiles were estimated by means of charts developed by the UK cross sectional reference data 1995 (Child Growth Foundation). Resuscitation was by means of intubation or cardiopulmonary resuscitation.

An episode of convulsions was defined as any form of convulsion, fit, seizure, or other turn in which consciousness was lost or any part of the body made abnormal movements.

An episode of lifelessness was defined as any episode in which the infant became lifeless.

A hospital admission was defined as any hospital admission or attendance including outpatients, excluding follow up from SCBU.

Illness in last week was defined as any illness, minor or major, diagnosed by a health professional in the last week.

Medication in the last week included treatment ointments, homeopathic medicine, and vitamins.

${ }^{\star}$ As reported by the parents.

†Few of these illnesses required hospitalisation.

CI, confidence interval; OR, odds ratio; SCBU, special care baby unit; SIDS, sudden infant death syndrome.

ratios, $95 \%$ confidence intervals, and $\mathrm{p}$ values were calculated taking into account the matching using the statistical package SAS. ${ }^{18}$ The same package was used to conduct conditional logistic regression for the multivariate analysis to compare each index group with matched controls. All appropriate variables significant at the $5 \%$ level in the univariate analysis were included in the modelling process. Models were constructed using the stepwise method for selection of variables: each non-significant variable was added to the final model to produce the appropriate relative statistic. Where the data have been partitioned (for example, for younger and older infants) and modelling techniques used the one to four ratio of index and controls has been preserved.

\section{Results}

ASCERTAINMENT

In the three year period there were over 470000 births in the study area and 456 unexpected infant deaths, of which 363 were classified as SIDS. Subsequent checking with statutory death registrations has identified a further eight deaths, not included in the study, which met the entry criteria (ascertainment 98.3\%). Of the 456 families, 30 refused an interview (93.4\% consent rate), 29 other families were excluded from the analysis because of police involvement (suspected non-accidental injury), because they lived outside the study regions, or because they could not be traced.

Our study deals with 325 SIDS deaths (90\% of the total SIDS deaths), 72 explained SUDI deaths $(77 \%$ of the total explained SUDI deaths), and four age matched controls for each index case (1588 controls).

The major causes of death among those considered by the panel to be fully explained were infection (46\%), accidental (15\%), congenital anomalies (14\%), and non-accidental injury $(13 \%)$. The remaining deaths included metabolic disorders, bowel obstruction, bronchopulmonary dysplasia, and cardiomyopathy.

INFANTS WHO DIED OF SIDS

Clinical characteristics were divided into those identifiable at the following times: birth, after discharge from hospital, during life, and shortly before death or the reference sleep. Table 1 shows the univariate findings. The data are from medical records unless otherwise stated. The most common neonatal problems included those related to preterm delivery, respiratory problems, jaundice, intrapartum asphyxia, metabolic problems, and minor infections. Major congenital anomalies were defined as those that were potentially life threatening if not treated, or those that would lead to severe disability. The most common major congenital anomalies include positional 
Table 2 Significant multivariate findings for clinical characteristics of SIDS cases and control infants

\begin{tabular}{|c|c|c|c|c|}
\hline & \multicolumn{2}{|c|}{ Model 1 Clinical characteristics only } & \multicolumn{2}{|c|}{ Model 2 Including other factors } \\
\hline & OR $(95 \% C I)$ & $p$ Value & OR $(95 \% C I)$ & $p$ Value \\
\hline \multicolumn{5}{|l|}{ At birth } \\
\hline$<10$ th birth weight centile & $2.02(1.23$ to 3.31$)$ & 0.006 & $2.44(1.13$ to 5.26$)$ & 0.02 \\
\hline$<37$ weeks' gestation & 2.07 (1.18 to 3.62$)$ & 0.01 & $4.93(2.16$ to 11.24$)$ & $<0.0001$ \\
\hline Twin or triplet & $5.83(1.86$ to 18.26$)$ & 0.003 & $7.81(1.35$ to 45.28$)$ & 0.02 \\
\hline Admission to SCBU & $2.12(1.26$ to 3.59$)$ & 0.005 & $1.25(0.53$ to 2.95$)$ & 0.62 \\
\hline Major congenital anomaly & $2.50(1.04$ to 6.01$)$ & 0.04 & $4.54(1.32$ to 15.56$)$ & 0.02 \\
\hline \multicolumn{5}{|l|}{ After hospital discharge } \\
\hline Problem with regurgitation & $2.53(1.35$ to 4.76$)$ & 0.004 & $1.52(0.55$ to 4.15$)$ & 0.42 \\
\hline \multicolumn{5}{|l|}{ During life } \\
\hline Episode of lifelessness & $3.72(1.99$ to 6.97$)$ & $<0.0001$ & $2.55(1.02$ to 6.41$)$ & 0.046 \\
\hline \multicolumn{5}{|l|}{ Before death/reference sleep } \\
\hline Last 24 hours: Baby Check (> 7) & $3.58(2.15$ to 5.95$)$ & $<0.0001$ & $4.17(1.88$ to 9.24$)$ & 0.0004 \\
\hline Demanded less feed & $3.48(1.45$ to 8.35$)$ & 0.005 & $1.89(0.41$ to 8.66$)$ & 0.42 \\
\hline
\end{tabular}

In model 2 the following factors were controlled for: sex, maternal age, parity, previous stillbirths and deaths, parental unemployment, maternal smoking during pregnancy, parental estimation of postnatal exposure to tobacco smoke, intention to breast feed, moving accommodation, recent maternal alcohol consumption, partner's use of illegal drugs, recent change in infant routine, put down in prone position, put down in side position, bed sharing, room sharing, duvet use, tog value of clothing and bedding, length of previous sleep, and infant found with head covered.

Centiles were checked by means of charts developed by the UK cross sectional reference data 1995 (Child Growth Foundation).

CI, confidence interval; OR, odds ratio; SIDS, sudden infant death syndrome; SCBU, special care baby unit.

deformities and malformations of both the genitourinary system and the cardiovascular system.

Infants who died of SIDS were significantly lighter for gestational age and had a shorter gestation. A small but significant proportion of SIDS cases were from multiple births. A greater proportion of infants who died of SIDS were unwell at or after birth (for example, needed resuscitating, were admitted to special care nursery, or had other neonatal problems). Although the rate of minor anomalies was not significantly different between cases and controls, significantly more of the infants who died of SIDS had major malformations, a third of which were cardiovascular. After discharge from hospital more infants who subsequently died of SIDS were noted by their parents to have problems with regurgitation, colic, poor weight gain, and other illnesses (the most common-found in over a third of both groups-being chest infection). Many of the infants on vitamin supplements were of short gestation, so the association of SIDS with vitamin supplementation became non-significant when controlled for gestational age.

During their short life, significantly more infants who died of SIDS experienced apparent life threatening events and more had hospital admissions. An apparent life threatening event was defined as an episode in which the infant became apparently lifeless. In the week before death, more infants who subsequently died of SIDS were diagnosed by health profes- sionals as having mainly minor illnesses or were thought to be in poor health by their parents.

In the 24 hours preceding death, a small but significant proportion of these infants were thought by their parents to be more sweaty than usual and not feeding as well. Although most infants who died of SIDS appeared well in the final 24 hours, over a fifth scored high enough on the Baby Check to suggest they needed assessment or advice from a health care professional or continued monitoring by parents.

Table 2 shows the multivariate models obtained when these clinical characteristics are modelled together (model 1) and when background associations are taken into account (model 2).

Many of the clinical characteristics that were significant in the univariate analysis were not significant when modelled together, and of those that were, some became non-significant when controlled for other significant factors associated with SIDS. A history of an apparent life threatening event among SIDS cases remained significant in the multivariate analysis at the 5\% level along with infants exhibiting birth weights below the 10 th centile, multiple births, and infants with major congenital anomalies. Taking a more conservative level of significance of $1 \%$, only prematurity and the Baby Check score remained as significant independent associations in the final model.

In the final 24 hours, significantly more of the infants who died of SIDS were showing

Table 3 Composite Baby Check score of SIDS cases and control infants in the last 24 hours

\begin{tabular}{|c|c|c|c|c|c|c|c|c|}
\hline \multirow[b]{2}{*}{ Score } & \multicolumn{2}{|l|}{ SIDS } & \multicolumn{2}{|l|}{ Controls } & \multirow{2}{*}{$\begin{array}{l}\text { Risk } \\
\text { ratio }\end{array}$} & \multirow[b]{2}{*}{ Unadjusted OR (95\% CI) } & \multirow[b]{2}{*}{ Age adjusted OR (95\% CI) } & \multirow[b]{2}{*}{ Multivariate OR (95\% CI) } \\
\hline & $n=318$ & $\%$ & $n=1299$ & $\%$ & & & & \\
\hline $0-7$ & 250 & 78.6 & 1202 & 92.5 & 0.85 & 1.00 (reference group) & 1.00 (reference group) & 1.00 (reference group) \\
\hline $8-12$ & 32 & 10.1 & 52 & 4.0 & 2.51 & $2.96(1.82$ to 4.80$)$ & $4.16(2.40$ to 7.23$)$ & $5.76(2.10$ to 15.78$)$ \\
\hline $13-19$ & 19 & 6.0 & 27 & 2.1 & 2.87 & $3.38(1.78$ to 6.42$)$ & $4.53(2.24$ to 9.13$)$ & $3.29(0.79$ to 13.69$)$ \\
\hline $20+$ & 17 & 5.3 & 18 & 1.4 & 3.86 & $4.54(2.20$ to 9.38$)$ & $3.82(1.75$ to 8.36$)$ & $2.06(0.38$ to 11.16$)$ \\
\hline
\end{tabular}

The risk ratio was calculated by dividing the prevalence of the SIDS score by the control score for each stratum.

In the multivariate analysis the following factors were controlled for: sex, maternal age, parity, previous stillbirths and deaths, parental unemployment, maternal smoking during pregnancy, parental estimation of postnatal exposure to tobacco smoke, intention to breast feed, moving accommodation, recent maternal alcohol consumption, partner's use of illegal drugs, recent change in infant routine, put down in prone position, put down in side position, bed sharing, room sharing, duvet use, tog value of clothing and bedding, length of previous sleep, and infant found with head covered.

No signs or symptoms were evident (Baby Check score $=0$ ) for $38.9 \%$ of SIDS cases and $59.4 \%$ of controls.

$\mathrm{CI}$, confidence interval; OR, odds ratio; SIDS, sudden infant death syndrome. 
Table 4 Composite Baby Check score of SIDS cases and control infants in the last 24 hours according to age

\begin{tabular}{|c|c|c|c|c|c|c|c|c|c|c|}
\hline \multirow[b]{3}{*}{ Score } & \multicolumn{5}{|c|}{ Younger infants (<101 days) } & \multicolumn{5}{|c|}{ Older infants (> 100 days) } \\
\hline & \multicolumn{2}{|c|}{ SIDS } & \multicolumn{2}{|c|}{ Controls } & \multirow{2}{*}{$\begin{array}{l}\text { Age adjusted } \\
\text { OR }(95 \% \text { CI) }\end{array}$} & \multicolumn{2}{|c|}{ SIDS } & \multicolumn{2}{|c|}{ Controls } & \multirow{2}{*}{$\begin{array}{l}\text { Age adjusted } \\
\text { OR }(95 \% \text { CI) }\end{array}$} \\
\hline & $n$ & $\%$ & $n$ & $\%$ & & $n$ & $\%$ & $n$ & $\%$ & \\
\hline $0-7$ & 137 & 81.1 & 654 & 94.0 & 1.00 (reference group) & 113 & 75.8 & 548 & 90.9 & 1.00 (reference group) \\
\hline $8-12$ & 18 & 10.7 & 19 & 2.7 & $7.20(2.98$ to 17.41$)$ & 14 & 9.4 & 33 & 5.5 & $2.61(1.25$ to 5.46$)$ \\
\hline $13-19$ & 11 & 6.5 & 13 & 1.9 & $4.87(1.74$ to 13.61$)$ & 8 & 5.4 & 14 & 2.3 & $3.51(1.31$ to 9.37$)$ \\
\hline $20+$ & 3 & 1.8 & 10 & 1.4 & $1.19(0.26$ to 5.41$)$ & 14 & 9.4 & 8 & 1.3 & $6.84(2.50$ to 18.71$)$ \\
\hline
\end{tabular}

Younger infants comprised 169 SIDS cases and 696 controls; older infants comprised 149 SIDS cases and 603 controls.

CI, confidence interval; OR, odds ratio; SIDS, sudden infant death syndrome.

signs and symptoms of illness to at least suggest they needed parental monitoring. This was the only variable in this time period to remain significant when controlled for other factors. Table 3 groups the scores according to the action that should be taken, as outlined previously.

Of those infants who scored more than 7 , three of the 15 individual signs and symptoms occurred in a significantly greater proportion of infants who died of SIDS than controls: these included infants being drowsy most of the time when awake $(14.9 \%$ v $2.0 \%$ controls; odds ratio $(\mathrm{OR})=8.51 ; 95 \%$ confidence interval (CI), 1.70 to 81.56$)$, infants wheezing ( $52 \%$ v $27 \%$ controls; OR $=3.00 ; 95 \% \mathrm{CI}, 1.49$ to 6.07), and infants taking less than half the normal amount of fluids in the last 24 hours $(39.7 \%$ v $29.5 \%$ controls; OR $=2.57$; $95 \% \mathrm{CI}$, 1.22 to 5.44$)$.

These data suggest a gradient of risk for those infants who scored higher, which is reflected in the ratio of risk between the SIDS cases and controls and the unadjusted OR. However, when the age matching is taken into account the gradient of risk is, if anything, in the opposite direction, suggesting an interaction with age. The decreasing gradient is even more evident in the multivariate results. Because the median age of all infants was 100 days, table 4 examines the risk associated with the Baby Check after splitting the data into those above and below this age.

Although the numbers are small in each cell the data suggest that the pattern of risk is different for the two age groups. For infants less than 101 days old, the increased risk appears to be among those infants with scores of 8-12, whereas for infants more than 100 days old, the risk increased with the score. A similar multivariate analysis to the one described above (table 2, model 2) for the two different age groups showed that the Baby Check scores were only significant for the younger infants scoring between 8 and 12 (multivariate
$\mathrm{OR}=20.50 ; 95 \% \mathrm{CI}, 4.03$ to 104.30$)$. If in the same age specific models we use a simple dichotomous variable representing the Baby Check score (healthy $v$ a score $>7$ ), this does not reach significance among the older infants $(\mathrm{OR}=2.49 ; 95 \%$ CI, 0.73 to 8.52 ) but remains significant among the younger infants $(\mathrm{OR}=10.77 ; 95 \% \mathrm{CI}, 2.98$ to 38.92$)$.

INFANTS WHO DIED OF EXPLAINED SUDI

In the univariate analysis, all the clinical features and health markers that were significant in the SIDS cases were in the same direction in the explained SUDI cases. Table 5 shows the results of conducting a similar multivariate analysis (but excluding factors related to the sleeping environment) for the infants who died of explained SUDI.

Unlike the infants who died of SIDS, neonatal problems and hospital admissions were significantly more common among the explained SUDI cases in the multivariate analysis, although hospital admissions became nonsignificant when controlled for other factors. The significance of neonatal problems is perhaps not surprising because $14 \%$ of these deaths were later explained by congenital anomalies. In both SIDS and explained SUDI deaths episodes of apparent life threatening events and a high Baby Check score in the 24 hours preceding death/reference sleep were much more frequent than in the controls.

Table 6 shows Baby Check scores for explained SUDI cases in relation to controls.

There were relatively few cases in some of the categories, so although there appears to be a progressive and substantial rise in the risk ratio for death with increasing Baby Check scores, the confidence intervals of the age adjusted odds ratio are wide. Only those scores greater than or equal to 20 are significantly greater than unity.

Table 5 Significant multivariate findings for clinical characteristics of explained SUDI cases and control infants

\begin{tabular}{|c|c|c|c|c|c|c|c|c|}
\hline & \multicolumn{2}{|c|}{ Explained SUDI } & \multicolumn{2}{|l|}{ Controls } & \multicolumn{2}{|c|}{ Model 1 Clinical features only } & \multicolumn{2}{|c|}{ Model 2 Including other factors } \\
\hline & $n$ & $\%$ & $n$ & $\%$ & OR $(95 \% C I)$ & p Value & OR $(95 \% C I)$ & p Value \\
\hline Neonatal problems & $26 / 69$ & 37.7 & $36 / 278$ & 12.9 & $3.83(1.29$ to 11.36$)$ & 0.02 & $4.64(1.34$ to 16.03$)$ & 0.02 \\
\hline Episode of lifelessness & $9 / 61$ & 14.8 & $10 / 288$ & 3.5 & 7.79 (1.59 to 38.12$)$ & 0.01 & $16.81(2.52$ to 112.3$)$ & 0.004 \\
\hline Hospital admission & $29 / 66$ & 43.9 & $43 / 288$ & 14.9 & $3.41(1.12$ to 10.36$)$ & 0.03 & $3.71(0.89$ to 15.47$)$ & 0.07 \\
\hline Baby Check (> 7) & $30 / 61$ & 49.2 & $22 / 288$ & 7.6 & $13.43(4.86$ to 37.15$)$ & $<0.0001$ & $31.20(6.93$ to 140.5$)$ & $<0.0001$ \\
\hline
\end{tabular}

In model 2 the following factors are controlled for: sex, maternal age, parity, previous stillbirths and deaths, parental unemployment, maternal smoking during pregnancy, parental estimation of postnatal exposure to tobacco smoke, intention to breast feed, moving accommodation, recent maternal alcohol consumption, partner's use of illegal drugs.

CI, confidence interval; OR, odds ratio; SUDI, sudden unexpected death in infancy. 
Table 6 Composite Baby Check score of explained SUDI cases and control infants in the last 24 hours

\begin{tabular}{|c|c|c|c|c|c|c|}
\hline \multirow[b]{2}{*}{ Score } & \multicolumn{2}{|c|}{ Explained SUDI } & \multicolumn{2}{|l|}{ Controls } & \multirow[b]{2}{*}{ Risk ratio } & \multirow[b]{2}{*}{ Age adjusted $O R(95 \% C I)$} \\
\hline & $n=61$ & $\%$ & $n=288$ & $\%$ & & \\
\hline $0-7$ & 31 & 50.8 & 266 & 92.4 & 0.55 & 1.00 (reference group) \\
\hline $8-12$ & 3 & 4.9 & 14 & 4.9 & 1.01 & $1.48(0.29$ to 7.45$)$ \\
\hline $13-19$ & 4 & 6.6 & 5 & 1.7 & 3.78 & $4.65(0.82$ to 26.26$)$ \\
\hline $20+$ & 23 & 37.7 & 3 & 1.0 & 36.20 & $63.21(8.70$ to 459.5$)$ \\
\hline
\end{tabular}

CI, confidence interval; OR, odds ratio; SUDI, sudden unexpected death in infancy.

SIDS CASES COMPARED WITH EXPLAINED SUDI CASES

In the multivariate analysis, a history of apparent life threatening disease or illness in the 24 hours preceding death measured by Baby Check remained significant for both groups of death. Figure 1 shows the number of episodes of apparent life threatening disease in the two index groups and the control group.

Figure 2 compares the Baby Check scores between the two index groups and the controls. Of the control infants a small proportion (7.5\%) had a Baby Check score higher than 7 in the 24 hours before the reference sleep; among the SIDS cases this proportion was three times higher (21.4\%), and among the explained SUDI cases the proportion was more than six times higher than the controls (49.2\%).

For those infants who scored between 8 and $12,34 \%$ of the parents of infants who died of SIDS and $47 \%$ of control parents thought their infant's health was good in the last 24 hours; among the three explained SUDI cases, none of the parents thought the infant was well. Of those infants who scored higher than $12,19 \%$ of both SIDS and control parents thought their infant was healthy, whereas $42 \%$ SIDS and $38 \%$ control parents did not contact a health professional, despite concerns about the infant's health. A much greater proportion of infants who subsequently died of explained

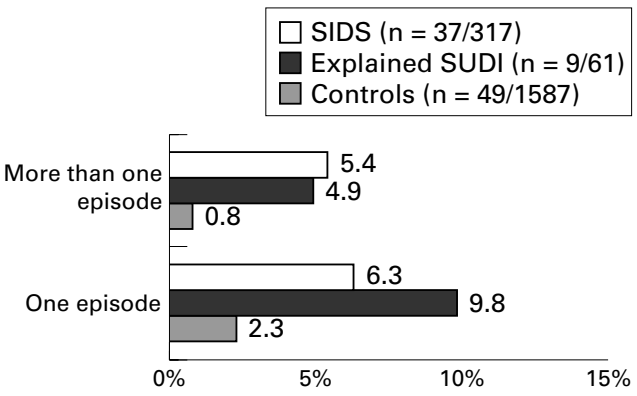

Figure 1 Frequency of episodes of lifelessness.

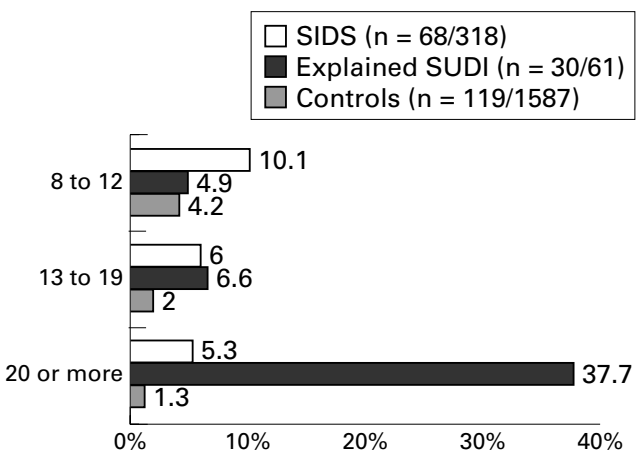

Figure 2 Frequency of Baby Check scores.
SUDI were identified as more severely ill (score $>19$ ) compared with the other two groups; consequently, of those infants who scored more than 12 , only $11 \%$ of parents thought their infant was healthy and the same proportion failed to contact a health professional.

\section{Discussion}

Our data demonstrate poorer health and a higher frequency of symptoms among infants dying suddenly and unexpectedly when compared with controls, both during life and shortly before death. This was in addition to the range of disadvantageous clinical characteristics identified antenatally, at birth, and after discharge from hospital. The data emphasise the importance of apparent life threatening diseases in this regard, and highlight the potential of the Baby Check score in helping to prevent at least some of these deaths.

The validity of data on reported health and illness depends heavily on accurate recall, and comparisons might be affected by differential recall among cases and controls. How confident are we that our results were not the result of recall bias? First, we attempted to build up a confidential and intimate relationship with the parents, contacting them through their own health visitor, and utilising experienced health care professionals to interview the parents in their own homes and facilitate bereavement care. Second, we ensured that all data were collected very soon after the death (for cases) and the reference sleep (for controls). Third, we sought not just parental opinion of infant health at birth or during life, but specific details from the parents of any medical contacts or hospitalisation to verify the severity of illness. Fourth, to measure infant health in the 24 hours before death/interview we used the Baby Check, which requires specific signs and symptoms and should therefore be less prone to recall bias. We cannot completely exclude the possibility of some recall bias, but the willingness and honesty with which parents answered some of the more intimate questions, the consistency of the findings over a range of clinical characteristics, and the proportional similarity of medical contacts and hospitalisation between the cases and controls suggest the steps we have taken has minimised the impact.

A history of an apparent life threatening disease was associated with deaths in both index groups, independently of poor infant health in the 24 hours preceding the death, when controlling for many other factors in the multivariate analysis. This emphasises the importance of apparent life threatening diseases as a marker of risk. About one in nine infants dying of SIDS, 
and one in seven explained deaths, experienced an episode of lifelessness compared with around one in 30 control infants. Of those babies with an apparent life threatening disease, $66 \%$ of SIDS cases, $67 \%$ of explained SUDI cases, and $67 \%$ of controls were brought to medical attention, but relatively more of the index infants (47\% SIDS, $44 \%$ explained SUDI v 33\% controls) were taken to hospital. If the rate of apparent life threatening diseases in controls is representative of the general population, as we believe, it suggests that in a population of 250000 with around 3000 deliveries a year there would be 100 infants a year experiencing an apparent life threatening diseases, of which 30 would arrive at hospital, but 30 would not be seen by a doctor at all.

Our results are consistent with our previous report, which highlighted that among the epidemiological characteristics only the age distribution, frequency of congenital malformations, and higher rates of smoking among mothers of SIDS cases were significantly different between infants who died of explained SUDI and those who died of SIDS. ${ }^{13}$ Several studies have looked at signs of illness preceding death and found specific symptoms among infants who died of SIDS, including respiratory problems, irritability, rash, change of cry, fever, ${ }^{19}$ gastrointestinal illness, ${ }^{19-22}$ and maternal reports of infants becoming sleepy, listless, and droopy. ${ }^{18}{ }^{19}$ Collectively, signs of illness before death appear to be more prevalent among infants who died of SIDS, although there appears to be no difference in major signs of illness ${ }^{10}$ or unreported severe illness..$^{93}$ Maternal descriptions of previous episodes of lifelessness ("held their breath", "stopped breathing") reported in the prospective Tasmanian study ${ }^{6}$ suggested an apparent life threatening event in the postnatal period. Similarly, events described by parents that were found to be significant in other studies include cyanotic attacks, ${ }^{24}$ convulsions, ${ }^{22}$ and tachypnoea, ${ }^{20}$ although this last finding disappears when controlled for gestational age and growth.

Our data are therefore consistent with other published work. However, the size of our study, which included virtually all the sudden deaths from a population of about half a million births, and the inclusion of deaths which, while unexpected, were subsequently explained, makes the findings more robust. We have been able to quantify, with much greater precision than previous studies, for both SIDS and explained SUDI, the characteristics of vulnerability arising in the neonatal period, and markers of the severity of illness in the 24 hours preceding death.

Although many of the epidemiological risk factors for SIDS (young maternal age, high parity, socioeconomic circumstance, etc) could be ascertained objectively from existing medical records, none was sufficiently specific to allow any particular intervention to be targeted at those infants most at risk of sudden death. In contrast, the Baby Check score is a means of quantifying acute illness, can be used by parents to help them decide whether to seek medical attention, and can be used by health

\section{Key messages}

- There are clinical features and health markers characterising increased vulnerability of infants who die suddenly and unexpectedly that are evident at birth, during life, and just before death

- These features, important among the infants with sudden infant death syndrome, are in the same direction among the explained sudden unexpected deaths

- Clinical features common to both groups of explained and unexplained deaths after controlling for possible confounders included a higher prevalence of an apparent life threatening event and ill health in the 24 hours before death

- "Baby Check" might help identify seriously ill babies at risk of sudden death, particularly in high risk infants

professionals as a triage tool to discriminate more reliably between those babies who should be assessed in hospital and those who need not. ${ }^{15}$ It measures the severity of the present illness and therefore can trigger interventions that could be life saving. In our study, we were obviously unable to accrue a prospective Baby Check score on those infants destined to die and therefore relied on recall (with the attendant problem of possible bias). We also needed to modify the score to reflect the circumstances of the data collection because we could not collect all possible dimensions of the Baby Check. Because the maximum possible modified score was 96, rather than 111, our retrospective score would tend to underestimate the baby's severity of illness. Although we used parental recognition of febrile illness as a proxy measure for a temperature reading, recent studies suggest that touch might overestimate a fever but would not fail to recognise it. ${ }^{25}$ On this measure, the score will therefore have overestimated the severity of illness for some infants, but the proportional difference between SIDS and control cases remained the same (Baby Check score > 7: fever included: $21.4 \%$ SIDS $v 7.6 \%$ controls; fever excluded: $18.2 \%$ SIDS $v 5.9 \%$ controls). Given the modifications to Baby Check made for our study and the possibility of recall bias it is difficult to derive a measure of specificity from the control infants. Concurrent assessment of the Baby $\mathrm{Check}^{26}$ suggests that our retrospective scoring system might have slightly overestimated the degree of illness, although the socioeconomic status of the 104 Cambridge mothers used in this field study was higher than the population norm. An accurate assessment of both the sensitivity and specificity of the Baby Check can only be made with a large scale population based intervention study.

According to the retrospective Baby Check score, over a fifth of infants who died of SIDS and nearly a half of the infants who died of explained SUDI were identifiably unwell in the 24 hours preceding death. Most of the infants who died of SIDS, especially the younger ones, displayed minor signs and symptoms, whereas 
for the explained deaths the signs and symptoms were potentially more serious. For both groups the evidence suggests that for a small proportion of infants showing signs of being severely unwell, appropriate action by parents or health care professionals might have prevented the death. This finding has important implications. Some studies have shown that a proportion of explained sudden unexpected deaths of infants, particularly those related to infections or to trauma, might be preventable. ${ }^{17} 2127-29$ It is well known from the series of CESDI reports that failure to recognise severity of illness is a recurring theme, and that this failure of recognition might not be only by the parents but by health professionals, who may give inappropriate reassurance that the baby is well. Although there are clear general messages for the necessity of better professional education and training, the use of the Baby Check score has the potential to guide less confident or experienced workers to "do the right thing". Concerns that the widespread use of Baby Check might result in a large extra burden on general practice appear to be unfounded. ${ }^{30} 31$

We are concerned at the level of discordance between the Baby Check score and the parental recognition of ill health in their baby, and the fact that this discordance occurred quite commonly in the control group as well as the index cases. Although many parents are good at recognising illness in their baby, some are less able, and others might not realise the importance of potentially serious signs such as subcostal recession. Many disadvantaged parents, particularly if young and of low educational achievement, might not be able to communicate their concerns effectively to health care professionals in a way that will convey the possible seriousness of the infant's condition. Baby Check, which has been carefully validated for use by doctors and parents, might allow parents to refine their judgement of an infant's degree of illness. Thus, there is the potential both to prevent "unnecessary" visits to the doctor, and to give parents more confidence in presenting their child when it is appropriate. Parental access to the Baby Check could be universal if it was routinely included in the "personal child health record".

These studies were supported by research grants from the national advisory body for the confidential enquiry into stillbirths and deaths in infancy (CESDI) and the Foundation for the Study of Infant Deaths (FSID).

1 CESDI. SUDI case control studies. Third annual report. London: Department of Health, 1996:55-77.

2 CESDI. Sudden unexpected deaths in infancy-the explained group. Fifth annual Report. London: Maternal and Child Health Research Consortium, 1998:25-39.

3 Blair PS, Fleming PJ, Bensley D, et al. Smoking and the sudden infant death syndrome: results of 1993-5 casecontrol study for confidential inquiry into stillbirths and deaths in infancy. BMF 1996;313:195-8.

4 Brooke H, Gibson A, Tappin D, Brown H. Case-control study of sudden infant death syndrome in Scotland, 19921995. BMF 1997;314:1516-20.

5 Mitchell EA, Ford RPK, Stewart AW, et al. Smoking and the sudden infant death syndrome. Pediatrics 1993;91:893-6.

6 McGlashan MD. Sudden deaths in Tasmania, 1980-1986: a seven year prospective study. Soc Sci Med 1989;29:1015seven

7 Bartholomew S, MacArthur BA. Comparison of infants dying from the sudden infant death syndrome with matched live controls. Soc Sci Med 1988;27:393-7.

\section{Appendix}

The Cambridge Baby Check

The Cambridge Baby Check is a scoring system to help parents and doctors quantify serious illness in babies up to 6 months of age. It is based on seven symptoms and 12 signs, each of which receives a score if they are evident, the higher the score the more ill the baby

Question Score

Have these symptoms been present in the last 24 hours?

(1) Has the baby vomited at least half the feed after each of the last three feeds?

(2) Has the baby had any bile stained (green) vomiting?

(3) Has the baby taken less fluids than usual in the last 24 hours? If so, score for the total amount of fluids taken as follows:

Taken slightly less than usual (more than $2 / 3$ normal)

Taken about half usual amount (1/3-2/3 normal)

Taken very little (less than $1 / 3$ normal)

(4) Has the baby passed less urine than usual?

(5) Has there been any frank blood (not streaks) 11 mixed with the baby's stools?

(6) Has the baby been drowsy (less alert than usual) when awake?

If so, score as follows:

Occasionally drowsy (but usually alert)

Drowsy most of the time (occasionally alert)

(7) Has the baby had an unusual cry (sounds unusual to mother)?

Now examine the baby awake

(8) Is the baby more floppy than you would 4 expect?

(9) Talk to the baby. Is the baby watching you $4^{\star}$ less than you expect?

(10) Is the baby wheezing (not snuffles or upper 3 respiratory noises) on expiration?

(11) Is the baby responding less than you would $5^{\star}$ expect to what is going on around?

Now examine the baby naked for the following checks

(12) Is there any indrawing (recession) of the lower ribs, sternum or upper abdomen? If so, score as follows:

Just visible with each breath?

Obvious and deep indrawing with each breath?

(13) Is the baby abnormally pale or has the baby looked very pale in the last 24 hours?

(14) Does the baby have blue fingernails or 3 toenails?

(15) Squeeze the big toe to make it white.

Release and observe colour for three seconds. Score if the toe is not pink within three seconds, or if it was completely white to start with?

(16) Has the baby got an inguinal hernia?

(17) Has the baby an obvious generalised trunkal rash or a sore and weeping rash covering an area greater than $5 \times 5 \mathrm{~cm}$ ?

(18) Is the baby's rectal temperature $38.3^{\circ} \mathrm{C}$ or more?

(19) Has the baby cried (more than just a grizzle) during this assessment?

$\star$ These questions were not asked.

†Because prospective temperature data could not be collected parents were asked whether their infant had recently had a fever. Copies of the Baby Check scoring system can be obtained from: Baby Check, PO Box 324, Wroxham, Norwich NR12 8EQ, UK. Telephone: +44 (0) 1603784400 .

8 Fedrick J. Sudden unexpected death in infants in the Oxford record linkage area. Details of pregnancy, delivery and abnormality in the infant. Br F Prev Soc Med 1974;28:16471.

9 Ford RPK, Mitchell EA, Stewart AW, et al. SIDS, illness and acute medical care. Arch Dis Child 1997;77:54-5.

10 Gilbert RE, Fleming PJ, Azaz Y, Rudd PT. Signs of illness preceding sudden unexpected death in infants. BMF 1990; 300:1237-9.

11 Cole TJ, Gilbert RE, Fleming PJ, Morley CJ, Rudd PT, Berry PJ. Baby Check and the Avon infant mortality study. Arry PJ. Baby Child 1991;66:1077-8.

12 Fleming PJ, Blair PS, Bacon C, et al. Environment of infants during sleep and risk of sudden infant death syndrome: 
results of $1993-5$ case-control study for confidential results of $1993-5$ case-control study for confidential inquiry into still

13 Leach CEA, Blair PP, Fleming PJ, et al. Sudden unexpected deaths in infancy: similarities and differences in the epidemiology of SIDS and explained deaths. Pediatrics 1999;104 e43.

14 Fleming PJ, Blair PS, Pollard K, et al. Pacifier use and SIDS - results from the CESDI SUDI case-control study. Arch Dis Child 1999;81:112-16.

15 Morley CJ, Thornton AJ, Cole TJ, Hewson PH, Fowler MA. Baby Check: a scoring system to grade the severity of acute systemic illness in babies under 6 months. Arch Dis Child 1991;66:100-6.

16 CESDI. Post mortem guidelines 1993. Second annual report - part 2. London: Department of Health, 1995: 120-1.

17 Gilbert R, Rudd P, Berry PJ, et al. Combined effect of infection and heavy wrapping on the risk of sudden unexpected infant death. Arch Dis Child 1992;67:171-7.

18 SAS Institute Inc. SAS statistical package. North Carolina, USA: SAS.

19 Carpenter RG, Gardner A, Pursall E, McWeeny PM. Identification of some infants at immediate risk of dying unexpectedly and justifying intensive study. Lancet 1979;ii:343346.

20 Hoffman HJ, Hillman LS. Epidemiology of the sudden infant death syndrome: maternal, neonatal, and postneonatal risk factors. Clin Perinatol 1992;19:717-37.

$21 \mathrm{Kahn}$ A, Blum D, Hennart P. A critical comparison of the history of sudden-death infants and infants hospitalised for near-miss for SIDS. Eur F Pediatr 1984;143:103-7.
22 Rintahaka PJ, Hirvonen J. The epidemiology of sudden infant death syndrome in Finland in 1969-80. Forensic Sci infant death syndrome

23 Clarke J, Davidson MM, Downham APS, et al. Newcastle survey of deaths in early childhood $1974 / 76$, with special reference to sudden unexpected deaths. Arch Dis Child 1977;52:828-35.

24 Cameron $\mathrm{MH}$, Williams AL. Development and testing of scoring systems for predicting infants with high-risk of sudden infant death syndrome in Melbourne. Aust Paediatr f 1986;(suppl):37-45.

25 Whybrew K, Murray M, Morley C. Diagnosing fever by touch: observational study. BMF 1998;317:321.

26 Thornton AJ, Morley CJ, Green SJ, Cole TJ, Walker KA, Bennett JM. Field trials of the Baby Check score card: mothers scoring their babies at home. Arch Dis Child 1991; 66:106-10.

27 Knowelden J, Keeling J, Nicholl JP. A multicentre study of post-neonatal mortality. London: HM Stationery Office, 1985.

28 Moore A. Preventable childhood deaths in Wolverhampton. BMF 1986;293:656-8.

29 Taylor EM, Emery JL. Family and community factors associated with infant deaths that might be preventable. BMF 1983;287:871-4.

30 Thompson H, Ross S, Wilson P, McConnachie A, Watson R. Randomised controlled trial of effect of Baby Check on use of health services in first 6 months of life. $B M \mathcal{F}$ 1999;318:1740-44.

31 Jewell D. Helping parents identify severe illnesses in their children. Editorial. BMF 1999;318:1711-22. 